\title{
The long-period companions of multiple stars tend to have moderate eccentricities
}

\author{
N. Shatsky^ \\ 1 Sternberg Astronomical Institute, Universitetskii pr. 13, Moscow 119899, Russia \\ 2 Royal Observatory of Belgium, Av. Circulaire 3, Bruxelles 1180, Belgium
}

Received 20 April 2001 / Accepted 2 October 2001

\begin{abstract}
We examined the statistics of an angle $\gamma$ between the radius vector of a visual companion of a multiple star and the vector of its apparent relative motion in the system. Its distribution $f(\gamma)$ is related to the orbital eccentricity distribution in the investigated sample. We found that for the wide physical subsystems of the 174 objects from the Multiple Star Catalogue $f(\gamma)$ is bell-shaped. The Monte-Carlo simulations have shown that our $f(\gamma)$ corresponds to the population of the moderate-eccentricity orbits and is not compatible with the linear distribution $f(e)=2 e$ which follows from stellar dynamics and seems to hold for wide binaries. This points to the absence of highly elongated orbits among the outer subsystems of multiple stars. The constraint of dynamical stability of triple systems is not sufficient to explain the "rounded-off" outer orbits; instead, we speculate that it can result from the angular momentum exchange in multiple systems during their early evolution.
\end{abstract}

Key words. binaries: visual - stars: statistics - formation

\section{Introduction}

The knowledge of the statistics of multiple star orbital and physical parameters is important for the studies of their formation processes. A variety of mechanisms invoked for modeling of the multiple star formation processes result in different shapes of the eccentricity distribution $f(e)$ and its dependence on the orbital period, which can be checked against the statistics of observed systems.

Visual double stars have been observed for many years mainly with the aim to determine stellar masses. Selection affects heavily the discovery and success of orbit computation for them (e.g. Ruymaekers 1999), hampering especially the study of highly inclined or eccentric orbits. Respective biases in statistics are very difficult to assess.

For the systems with periods $P>500-1000$ years, the computation of orbits becomes almost impossible and hence there is no way to obtain $f(e)$ directly. Meanwhile, bound systems with periods up to $10^{5}-10^{6}$ years do exist (e.g. Palasi 2000), so more than three orders of magnitude in period escape from the study of orbital parameter statistics.

Tokovinin (1998) proposed to determine the shape of $f(e)$ for wide visual binaries from the distribution of the angle $\gamma$ between the radius vector of a secondary

\footnotetext{
* e-mail: kolja@sai.msu.ru
}

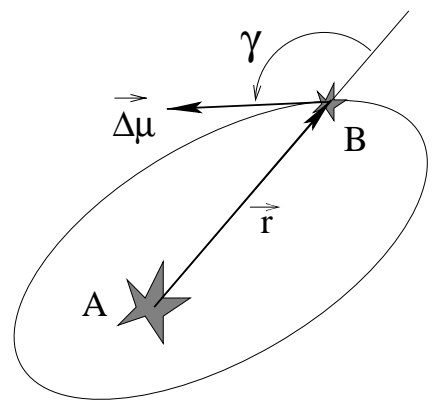

Fig. 1. Definition of angle $\gamma$ for the motion of the star B in the system $\mathrm{A}-\mathrm{B}$. In the sky plane, radius vector $r$ connects the gravity centers of $\mathrm{A}$ and $\mathrm{B} ; \Delta \mu$ shows the direction of the apparent relative motion of $\mathrm{B}$ with respect to A.

companion and the vector of its apparent relative motion in the system (Fig. 1). For a sample of Hipparcos binaries studied by Brosche et al. (1992, BDS92), such a distribution $f(\gamma)$ is approximately flat. According to Tokovinin (1998), this corresponds to the linear distribution of eccentricities, $f(e)=2 e$ predicted by stellar dynamics (Ambartsumian 1937). Duquennoy \& Mayor (1991) affirm that $f(e)=2 e$ applies to the visual binaries in the solar neighborhood. However, as we show below, this is not the case for multiple systems.

With the new data from Hipparcos and the database of the Multiple Star Catalogue (MSC, Tokovinin 1997) we address the question about the $f(e)$ for long-period outer subsystems of multiple $(N>2)$ stars. MSC is a unique collection of high-order systems which are confirmed to be physical by a combination of several criteria. 
Our basic idea is to obtain some constraints on $f(e)$ for this previously unstudied domain of long periods dealing with definitely non-optical pairs. Also, it is intriguing to test whether the multiple $(N>2)$ stars differ in orbital characteristics from the whole ensemble of non-single objects, most of which contain, apparently, only two stars.

\section{Sample selection and astrometric data}

For our analysis we selected from the MSC database the outer subsystems of hierarchical multiple stars with periods from 500 to $10^{6}$ years. These periods were inferred from the projected separation $\rho$, the parallax and the total mass of the object. The physical status of these visual components is established by at least two independent criteria (such as the position at the colour-magnitude diagram, common proper motions or common radial velocities). We limited the study to primary components less massive than $5 M_{\odot}$. Too distant systems with parallax $\pi<0$.'003 were not considered either. This gave an initial sample of 376 systems.

The vectors of the relative motion of the secondary outer components were derived from the change of position parameters $\rho$ and $\theta$ relative to the primary star between the first and the last observations of the system after correction for the precession. The first-epoch data were taken mainly from the Washington Double Star catalogue (Worley \& Douglass 1996). In few cases the positions given in the CCDM (Dommanget \& Nys 1994) were used (flag "c" in Table 1, see below).

To get the most precise second epoch positions, we identified the components of our objects in the Hipparcos (ESA 1997) and Tycho-II (Høg et al. 2000) catalogues. The confusions in identification between different catalogues were resolved with the help of Digital Sky Survey images. For four systems in the output sample (flag "** in Table 1), the alternative solutions were accepted as given in Hipparcos Notes (HIP 17749/50, 24019/20), by Falin \& Mignard (1999; HIP 64425) or Fabricius \& Makarov (2000; HIP 76563/66). The identifications of components in WDS, CCDM, Hipparcos and Tycho catalogues are given in Table $2^{1}$. Only a small fraction of objects with faint outer companions have no Hipparcos positions (flag "w" in Table 1).

For twelve systems the difference of Tycho-II proper motions appeared to be more reliable for the determination of $\gamma$ than the change of the $\rho, \theta$ data (flag " $m$ " in Table 1).

Some companions of visual multiple stars which were treated as photocenters in WDS (e.g. systems like "AB-C" in WDS) were resolved in Hipparcos observations. To compare consistently the position parameters provided by WDS and Hipparcos in this case, we computed the

\footnotetext{
1 Table 2 is only available in electronic form at the CDS via anonymous ftp to cdsarc.u-strasbg.fr (130.79.125.5) or via

http://cdsweb.u-strasbg.fr/cgi-bin/qcat?J/A+A/380/238
}

positions of the photocenter of inner subsystems for the second epoch using the $V_{\mathrm{T}^{-}}$or $H p$-band fluxes for weighting.

\section{Angle $\gamma$ and the role of subsystems}

The vectors of the outer component relative motion (modulus $\delta$ and the angle $\gamma$ ) were computed as follows:

$\delta^{2}=\rho_{1}^{2}+\rho_{2}^{2}-2 \rho_{1} \rho_{2} \cos \Delta \theta$

$\tan \gamma=\frac{\rho_{2} \sin \Delta \theta}{\rho_{2} \cos \Delta \theta-\rho_{1}}$

where $\Delta \theta=\theta_{2}-\theta_{1}$ and indices 1 and 2 refer to the first and second epoch observations, respectively. The angle $\gamma$ refers hereby to the second epoch. The errors of $\delta$ and $\gamma$ come mostly from the uncertainty of the WDSposition which is poorly defined for visual observations. Nevertheless, we adopt the error model based on the works of Douglass (1992) and Pannunzio et al. (1988). The error in separation $\epsilon_{\rho}$ grows linearly from $\pm 00^{\prime \prime} 07$ to $\pm 00^{\prime \prime} 3$ in the $0^{\prime \prime}-30^{\prime \prime}$ range of $\rho$. The transversal error $\rho \epsilon_{\theta}$ grows from a 0.5 to 1.0 fraction of this value in the same range. For systems wider than $30^{\prime \prime}$, the error of $\theta$ is dominated by the $\pm 0.5^{\circ}$ round-off error of the published WDS catalogue data.

In a hierarchical multiple star, the outer companion orbits around the center of mass of stars which constitute the inner hierarchical levels. This means that the body "A" (and/or "B") in Fig. 1 is in fact the gravity center of inner sub-systems. Meanwhile, the measurements are made having as a reference point either the photocenter or even one of the companions of an inner subsystem (if it is resolved). This reference point also shifts at the sky due to the orbital motion in the inner subsystem. A correction of the position parameters is needed to convert the observed parameters $\rho, \theta$ into $\rho_{0}, \theta_{0}$, latter referring already to the position of the gravity center of the inner subsystem.

This correction was applied in case of the known orbital motion in the inner subsystem. Otherwise, an estimate of the unknown photocenter motion in inner subsystems was incorporated into the error assessment of the calculated $\gamma$. This procedure removed from the sample the systems in which an apparent motion of the outer companion reflects mainly the orbital motion in the inner subsystem.

The final values of the relative motion modulus $\delta$ were checked to be consistent with the expected orbital motion velocities. A few $\delta$ appear unreally large and mostly result from uncertainty of a position angle. Nevertheless, respective dynamical parallaxes

$D P=0^{\prime \prime} 418\left[\frac{\rho}{\operatorname{Mass}_{\mathrm{sys}}^{\Sigma}}\left(\frac{\delta}{\Delta(\text { Epoch })}\right)^{2}\right]^{1 / 3}$

(Russell \& Moore 1940) statistically resemble the MSC parallax estimates $\pi$ : median ratio $D P / \pi$ is $1.15 ; 10 \%$ and $90 \%$-percentiles of this ratio are 0.67 and 3.0. 
The values of the $\gamma$-angle corrected for motion in the inner subsystems are given in Table 1. In total, 174 systems are presented for which all the corrections still allow to determine $\gamma$ with a precision better than $\pm 30^{\circ}$. The note column provides the information on the used sources of data and on the applied corrections for the calculation of $\gamma$.

The relative proper motions derived by us suffer to some extent from round-off errors in WDS data for the first epoch positions. In contrast to our approach, Brosche \& Sinachopoulos $(1988,1989)$ used original observational data to compute the relative motions instead of somehow generalized values in WDS. Nevertheless, the fifteen objects which are common to both studies show the good agreement of the derived angles $\gamma$ given our $\gamma$-errors $\epsilon_{\gamma}$ :

$\left\langle\left|\gamma_{\mathrm{BS}}-\gamma\right| / \epsilon_{\gamma}\right\rangle \approx 1.0$.

It is worth to note that the usage of precise second-epoch parameters removes some biasing of angles $\gamma$ caused by the round-off errors (this bias would be noticeable if only the WDS data were used).

\section{Distribution of $\gamma$ and its simulation}

The distribution $f(\gamma)$ is shown in the Fig. 2 as a thick line. The negative and positive values of $\gamma$ are folded into a $0^{\circ}-180^{\circ}$ interval. The bin size is $30^{\circ}$ given the $\epsilon_{\gamma}$ range $0-30^{\circ}$. In contrast to the uniform distribution obtained by BDS92, our $f(\gamma)$ is bell-shaped. The difference from the uniform distribution of $\gamma$ is significant at the level of $99.95 \%$ so the corresponding $f(e)=2 e$ does not fit our data.

Although our data are limited (only 6 bins in the histogram), we performed some Monte-Carlo simulations to restore the possible shape of $f(e)$. For this, an artificial set of $N=10000$ binaries was generated using the software provided by A. Tokovinin. The following types of the distribution of $e$ were tested: fixed $e=$ const., linear $f(e)=2 e$ (with eccentricity cut-off: $e<e^{*}$ ) and bell-like $f(e)=(\pi / 2) \sin (\pi e)$. The orbits had random orientation and phase. Then the resulting angles $\gamma$ and their histograms $f(\gamma)$ were computed. The criterion $\chi^{2}$ was used to reject the distributions of $\gamma$ which are not compatible with our data.

As a result (see plotted curves in Fig. 2), the good fit was obtained for any artificial sample with single eccentricity $e<0.5$. At the 99\%-level of significance all the single $e>0.6$ are rejected. The linear relation $f(e)=2 e$ does not fit our data for the cutoff values $e^{*}>0.85$ at the same level of significance. Meanwhile, the uniform and bell-shaped distributions of eccentricities give an $f(\gamma)$ very similar to the observed one. In general, all the distributions populated mostly by low-to-moderate values of eccentricity cannot be rejected.

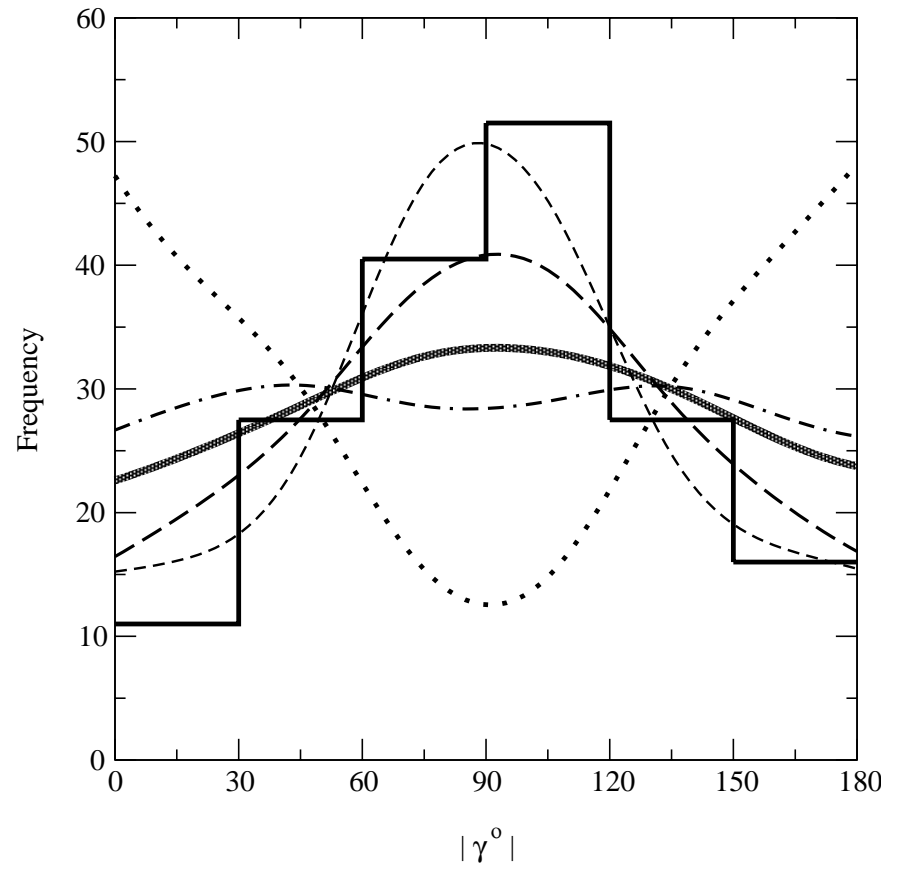

Fig. 2. Distribution $f(\gamma)$ for $174 \mathrm{MSC}$ wide subsystems whose movement is still significant after a correction for internal movement was applied, i.e. $\epsilon_{\gamma}<30^{\circ}$. Results of Monte-Carlo simulations are also shown. Designations: Stair-case: observed $f(\gamma)$; short- and long-dashed lines are for $e=0.4 \quad\left(\chi^{2}=4\right)$ and bell-shaped distribution $f(e) \propto \sin (\pi e) \quad\left(\chi^{2}=8\right)$. Incompatible distributions: dotted line for high-e orbits $(e=0.9$ is shown; $\left.\chi^{2}=177\right)$; dot-dashed and grey thick lines - for linear $f(e)=2 e$ with cutoff $e^{*}=0.99 \quad\left(\chi^{2}=38\right)$ and with stability-constrained rejection $\left(\chi^{2}=23\right.$, see Discussion $)$.

\section{Discussion}

Some characteristics of the final sample are shown in Fig. 3. As expected, the shortest periods are most frequent, although the periods as long as $\sim 10^{5} \mathrm{yr}$ are present (for nearby systems). The median period is $6100 \mathrm{yr}$. Having a characteristic separation of $\sim 10^{\prime \prime}$ and bestrepresented first epoch of 1835 , the system with such a period gives a motion of $\sim 1.4^{\prime \prime}$ in 155 years. As we see, there is nothing mysterious about detecting the orbital motion at such long periods.

The constraint accepted for maximal stellar mass in the system seems reasonable: there are few cases of $M_{\max }^{*}>4 M_{\odot}$. So, our study is restricted to low-tointermediate mass stars.

The difference of our $\gamma$-angle distribution from a uniform one is clearly seen. It is unlikely that this result is heavily distorted by selection. The errors of the measurements of the position angle are at least not larger than in separation (Pannunzio et al. 1988) so the excess of values near $90^{\circ}$ is hard to explain in this way. Both our and BDS92's samples refer roughly to the same range of periods but, apparently, differ in fraction of highly elongated orbits. 

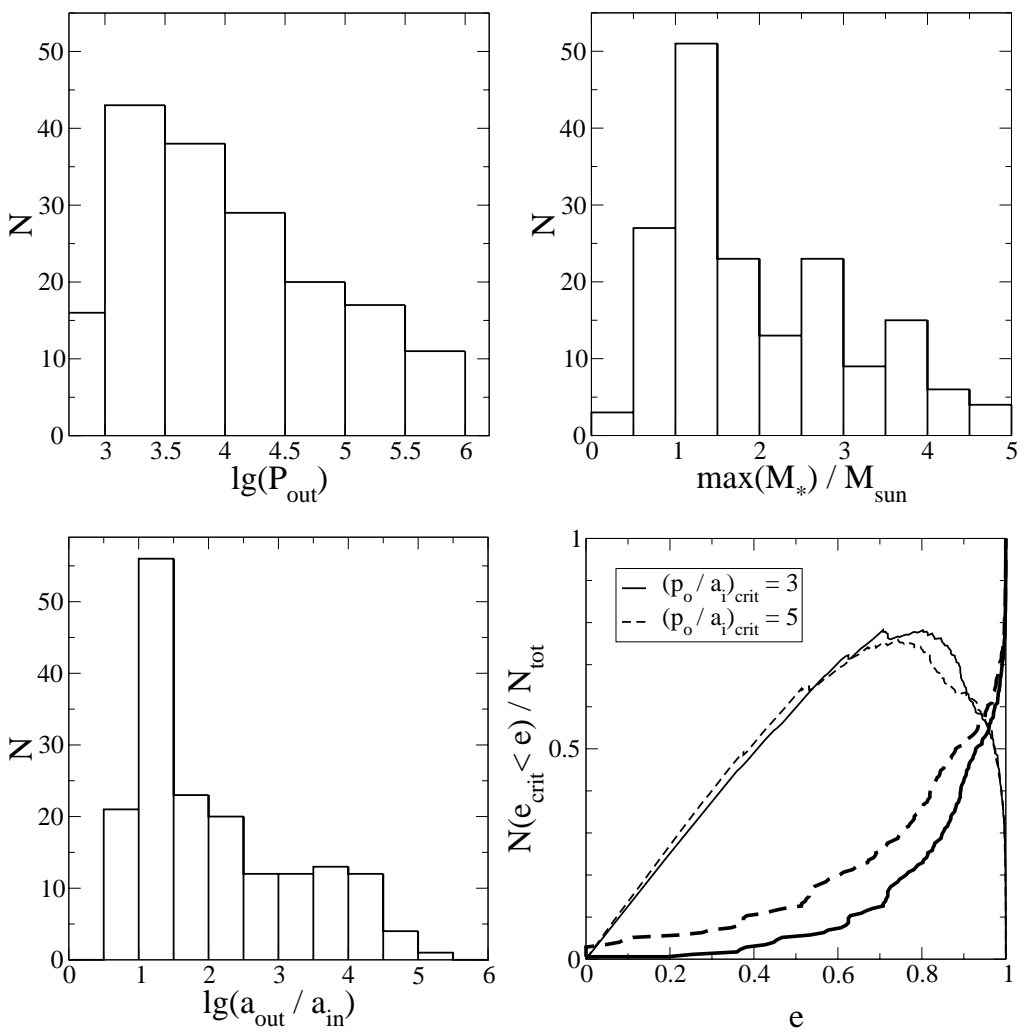

Fig. 3. Final sample characteristics: Left: the histogram of the logarithm of outer subsystem period; Right: the distribution of the mass of the most massive star in the system.
For visual orbits, a bell-shaped distribution of eccentricity was obtained by Ruymaekers (1999). Nevertheless, the author notices (after Harrington 1977b) that in orbit computation the quality of inclined or eccentric orbits is strongly degraded and that probably for this reason good high-e orbits are rare. It is obviously not the case for objects in our sample with periods longer than $500-1000$ years. Since the position changes are mostly very small compared to the separation, the systems with large eccentricities are not discriminated to be discovered or measured.

Still keeping in mind the possibility of some selection in the MSC itself, we nevertheless suggest that eccentric long-period orbits among outer subsystems in multiple stars are rare. Apart from some possible unaccounted systematic errors in observations, two explanations can be advanced for our finding.

Dynamical instability. Our systems are all hierarchical: they have the size of the outer subsystem at least several times wider than that of inner subsystems (Fig. 4). To check whether the dynamical instability factor is responsible for "damping" of high eccentricities in our $f(e)$, we can apply the crude criterion of stability for the ratio of the outer subsystem periastron distance $R_{\mathrm{p}}^{\text {out }}$ to the size of an inner orbit $a_{\text {in }}$ :

$\frac{R_{\mathrm{p}}^{\text {out }}}{a_{\text {in }}} \approx \frac{\rho_{\text {out }}\left(1-e_{\text {out }}\right)}{\rho_{\text {in }}}>C$

where $C$ is about $3-5$ and, in principle, is a weak function of eccentricity $e_{\text {out }}$ and mass ratio $q_{\text {out }}$ (Harrington 1977a). In high-order $(N>3)$ multiples, $a_{\text {in }}$ stands for the size of

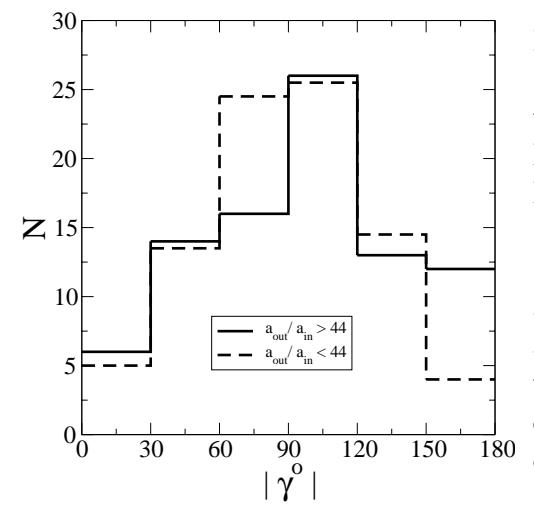

Fig. 5. Distributions $f(\gamma)$ for two halves of the sample: with $a_{\text {out }} / a_{\text {in }}$ less than 44 (dashed line) and more than 44 (solid line). Deviations of $f(\gamma)$ from flat are characterized by $\chi^{2} \approx 29$ and $\chi^{2} \approx 15$, respectively; this tendency is expected from stability constraints.

the widest subsystem (Mardling \& Aarseth 2001, p. 414). Here we suppose that, statistically, the semi-major axis $a$ of an orbit is close to the observed projected separation $\rho$.

For a given $C$, we can derive some critical value of the eccentricity $e_{\text {crit }}$ for each our system which would "make" it marginally stable. The cumulative distributions $F$ of these $e_{\text {crit }}$ are shown in Fig. 4 (right). If we assume that the universal $f(e)=2 e$ distribution applies to all multiple systems and that the highest eccentricities are eliminated by instability, then the modified distribution can be modeled as $f(e)=2 e \cdot[1-F(e)]$, where $F(e)$ is the cumulative distribution of critical eccentricities (thin lines in the same figure).

We reran our Monte-Carlo simulations with the modified $f(e)$, noticing the weak sensitivity of this $f(e)$ to the value of $C$. As expected, the resulting $f(\gamma)$ is a bit closer to the observed one (thick grey line in Fig. 2), but still far from it.

Alternatively, we can try to look for the variation of $f(\gamma)$ among the subsamples with different $a_{\text {out }} / a_{\text {in }}$. 
Table 1. Angle $\gamma$ for objects of an output sample. Columns are: WDS-designation of the object; outer subsystem identification as given in MSC; second epoch separation and position angle; angle $\gamma$ and its error; motion $\delta^{\prime \prime}=\Delta \mu\left(T_{2}-T_{1}\right)$ between two epochs; note symbols (see bottom of the table). Values $\gamma$ and $\delta$ are corrected for the motion in the subsystems, if it is significant.

\begin{tabular}{|c|c|c|c|c|c|c|c|c|c|c|c|c|c|c|c|}
\hline WDS & SYS & $\rho_{2}{ }^{\prime \prime}$ & $\theta_{2}^{\circ}$ & $\gamma^{\circ}$ & $\epsilon_{\gamma}{ }^{\circ}$ & $\delta^{\prime \prime}$ & note & WDS & SYS & $\rho_{2}{ }^{\prime \prime}$ & $\theta_{2}^{\circ}$ & $\gamma^{\circ}$ & $\epsilon_{\gamma}{ }^{\circ}$ & $\delta^{\prime \prime}$ & note \\
\hline $00057+4549$ & $\mathrm{AF}$ & 328.0 & 254 & 215 & 24 & 1.6 & $\mathrm{cO}$ & $07185-5721$ & $\mathrm{AB}$ & 2.4 & 254 & 97 & 13 & 0.6 & $\mathrm{w}$ \\
\hline $00108-5729$ & $\mathrm{AB}$ & 3.2 & 183 & 108 & 16 & 0.3 & & $07204-5219$ & $\mathrm{AB}$ & 9.2 & 26 & 128 & 5 & 1.6 & \\
\hline $00134+2659$ & $\mathrm{AC}$ & 18.0 & 225 & 0 & 24 & 0.6 & $\mathrm{wX}$ & $07359+4302$ & $\mathrm{AB}$ & 2.1 & 88 & 32 & 14 & 0.3 & \\
\hline $00150+0849$ & $\mathrm{AB}$ & 11.5 & 148 & 264 & 18 & 0.5 & & $07378-0236$ & $\mathrm{AC}$ & 1.4 & 303 & 31 & 6 & 0.6 & 1 \\
\hline $00174+0853$ & $\mathrm{BC}$ & 4.0 & 235 & 195 & 14 & 0.3 & 1 & $07354-7417$ & $\mathrm{AB}$ & 1.9 & 125 & 109 & 6 & 0.8 & \\
\hline $00219-2300$ & $\mathrm{AB}$ & 6.1 & 26 & 93 & 10 & 0.7 & 2 & $08047+4717$ & $\mathrm{AC}$ & 5.9 & 214 & 303 & 15 & 0.2 & $\mathrm{~m}$ \\
\hline $00321-0511$ & $\mathrm{AC}$ & 2.6 & 190 & 283 & 6 & 1.0 & 1 & $08044+1217$ & $\mathrm{AC}$ & 5.0 & 213 & 99 & 4 & 2.3 & w1 \\
\hline $00335-5520$ & $\mathrm{AC}$ & 6.8 & 245 & 235 & 22 & 0.3 & 10 & $08065-0915$ & $\mathrm{AB}$ & 30.6 & 327 & 132 & 22 & 0.4 & $\mathrm{mx}$ \\
\hline $00345-0433$ & $\mathrm{AC}$ & 19.7 & 45 & 194 & 24 & 0.5 & 10 & $08198-7131$ & $\mathrm{AB}$ & 64.8 & 58 & 263 & 11 & 1.6 & \\
\hline $00360+2959$ & $\mathrm{AB}$ & 6.2 & 23 & 299 & 4 & 1.5 & & $08267+2432$ & $\mathrm{AB}$ & 5.7 & 50 & 104 & 5 & 1.2 & \\
\hline $00393+3052$ & $\mathrm{AB}$ & 28.7 & 298 & 327 & 24 & 1.0 & $\mathrm{w}$ & $08368+7443$ & $\mathrm{AB}$ & 1.5 & 234 & 191 & 13 & 0.3 & \\
\hline $00493-2124$ & $\mathrm{AC}$ & 11.0 & 299 & 342 & 13 & 0.1 & $\mathrm{mX}$ & $08555-0758$ & $\mathrm{AB}$ & 4.1 & 3 & 130 & 8 & 0.7 & \\
\hline $01041+2635$ & $\mathrm{AB}$ & 0.8 & 244 & 5 & 23 & 0.1 & & $09125-4337$ & $\mathrm{AB}$ & 2.8 & 282 & 270 & 20 & 0.3 & \\
\hline $01137+0735$ & $\mathrm{AB}$ & 22.9 & 63 & 216 & 19 & 0.9 & $\mathrm{x}$ & $09205-0933$ & $\mathrm{AB}$ & 229.1 & 211 & 262 & 11 & 2.2 & \\
\hline $01158-6853$ & $\mathrm{AC}$ & 319.3 & 310 & 106 & 9 & 3.3 & $2 \mathrm{O}$ & $09272-0913$ & $\mathrm{AC}$ & 10.5 & 176 & 174 & 24 & 0.3 & 1 \\
\hline $01230-1258$ & $\mathrm{AB}$ & 40.5 & 313 & 216 & 13 & 0.2 & mo & $09354+3958$ & $\mathrm{AB}$ & 25.0 & 149 & 72 & 24 & 0.6 & \\
\hline $01409+4952$ & $\mathrm{AC}$ & 6.1 & 282 & 34 & 26 & 0.2 & & $10311-2411$ & $\mathrm{AB}$ & 1.9 & 67 & 352 & 24 & 0.2 & \\
\hline $01413+2545$ & $\mathrm{AB}$ & 10.5 & 31 & 197 & 12 & 0.8 & $\mathrm{w}$ & $10350+0839$ & $\mathrm{AB}$ & 2.2 & 157 & 217 & 15 & 0.3 & \\
\hline $01493+4754$ & $\mathrm{AC}$ & 20.8 & 178 & 303 & 26 & 0.5 & $\mathrm{wO}$ & $10401+1914$ & $\mathrm{AC}$ & 6.4 & 355 & 49 & 29 & 0.3 & $\mathrm{O}$ \\
\hline $01551+2847$ & $\mathrm{AC}$ & 5.7 & 163 & 272 & 17 & 0.4 & 10 & $10409-3545$ & $\mathrm{AB}$ & 0.6 & 48 & 236 & 23 & 0.2 & \\
\hline $01590-2255$ & $\mathrm{AB}$ & 8.6 & 303 & 301 & 24 & 0.3 & & $10435+4612$ & $\mathrm{AB}$ & 288.0 & 88 & 267 & 11 & 1.7 & \\
\hline $01586-5332$ & $\mathrm{AB}$ & 2.3 & 103 & 306 & 17 & 0.3 & & $10443-7052$ & $\mathrm{AB}$ & 62.7 & 75 & 250 & 21 & 1.0 & $\mathrm{x}$ \\
\hline $02128+7941$ & $\mathrm{AB}$ & 55.6 & 277 & 263 & 12 & 1.4 & & $10575-1105$ & $\mathrm{AC}$ & 4.5 & 8 & 91 & 13 & 0.5 & 1 \\
\hline $02124+3018$ & $\mathrm{AB}$ & 3.9 & 70 & 274 & 8 & 0.7 & & $11047-0413$ & $\mathrm{AB}$ & 11.6 & 221 & 60 & 5 & 2.0 & 2 \\
\hline $02110-3540$ & $\mathrm{AC}$ & 13.6 & 41 & 236 & 18 & 1.0 & $\mathrm{O}$ & $11170-0708$ & $\mathrm{AB}$ & 1.0 & 210 & 229 & 16 & 0.4 & $\mathrm{w}$ \\
\hline $02128-0224$ & $\mathrm{AB}$ & 16.6 & 234 & 57 & 6 & 1.9 & & $11366+5608$ & $\mathrm{AB}$ & 6.1 & 166 & 326 & 6 & 0.9 & \\
\hline $02280-5808$ & $\mathrm{AC}$ & 17.6 & 299 & 264 & 26 & 0.3 & $\mathrm{O}$ & $11387+4507$ & $\mathrm{AB}$ & 9.2 & 250 & 238 & 3 & 3.1 & \\
\hline $02370+2439$ & $\mathrm{AB}$ & 38.2 & 275 & 127 & 25 & 0.7 & & $12143+1149$ & $\mathrm{AB}$ & 1.7 & 16 & 359 & 16 & 0.4 & \\
\hline $02411+1848$ & $\mathrm{AC}$ & 65.5 & 242 & 61 & 12 & 0.4 & $\mathrm{~m}$ & $12413-1301$ & $\mathrm{AB}$ & 5.3 & 312 & 99 & 5 & 1.2 & \\
\hline $02529+5300$ & $\mathrm{AC}$ & 1.6 & 312 & 73 & 10 & 0.5 & 1 & $12492+8325$ & $\mathrm{AB}$ & 21.5 & 326 & 130 & 16 & 0.8 & \\
\hline $02583-4018$ & $\mathrm{AB}$ & 8.3 & 90 & 93 & 7 & 1.1 & & $12563+5406$ & $\mathrm{AB}$ & 3.8 & 281 & 313 & 8 & 0.7 & \\
\hline $03158+5057$ & $\mathrm{AB}$ & 1.5 & 102 & 5 & 4 & 0.9 & & $13066-6434$ & $\mathrm{AB}$ & 0.5 & 234 & 154 & 13 & 0.3 & \\
\hline $03302+5922$ & $\mathrm{AB}$ & 2.6 & 70 & 120 & 20 & 0.4 & $\mathrm{X}$ & $13123-5955$ & $\mathrm{AC}$ & 1.9 & 7 & 84 & 7 & 0.7 & $1^{*}$ \\
\hline $03480+6840$ & $\mathrm{AB}$ & 17.1 & 14 & 272 & 12 & 0.9 & $2^{*}$ & $13145-2417$ & $\mathrm{AC}$ & 12.5 & 332 & 282 & 22 & 0.5 & 1 \\
\hline $03470+4126$ & $\mathrm{AB}$ & 7.3 & 54 & 139 & 3 & 2.1 & & $13196+3507$ & $\mathrm{AB}$ & 17.6 & 129 & 90 & 5 & 2.9 & \\
\hline $03566+5042$ & $\mathrm{AB}$ & 75.3 & 31 & 270 & 22 & 0.8 & & $13258+4430$ & $\mathrm{AC}$ & 2.6 & 205 & 79 & 6 & 1.2 & $\mathrm{w}$ \\
\hline $04226+2538$ & $\mathrm{AB}$ & 19.4 & 25 & 263 & 28 & 0.5 & & $13328-1746$ & $\mathrm{AB}$ & 1.2 & 26 & 326 & 12 & 0.3 & \\
\hline $04436-0848$ & $\mathrm{AB}$ & 9.3 & 318 & 316 & 27 & 0.3 & & $13437-4204$ & $\mathrm{AC}$ & 0.9 & 72 & 40 & 24 & 0.2 & 1 \\
\hline $04475+4324$ & $\mathrm{AC}$ & 1.4 & 24 & 270 & 14 & 0.4 & 1 & $14135+5147$ & $\mathrm{AB}$ & 13.5 & 236 & 339 & 8 & 1.0 & \\
\hline $04563+5206$ & $\mathrm{AC}$ & 4.6 & 114 & 341 & 7 & 0.7 & 1 & $14158+1018$ & $\mathrm{AC}$ & 3.9 & 148 & 312 & 7 & 0.7 & 1 \\
\hline $05012+3430$ & $\mathrm{AB}$ & 1.7 & 229 & 339 & 19 & 0.2 & & $14182-2731$ & $\mathrm{AC}$ & 3.3 & 111 & 284 & 17 & 0.3 & $1 \mathrm{x}$ \\
\hline $05017+2640$ & $\mathrm{AC}$ & 78.4 & 160 & 248 & 26 & 0.8 & 1 & $14234+0827$ & $\mathrm{AB}$ & 6.3 & 194 & 85 & 7 & 0.9 & \\
\hline $05098+2802$ & $\mathrm{AB}$ & 11.5 & 28 & 181 & 26 & 0.3 & $2^{*}$ & $14375+4743$ & $\mathrm{AC}$ & 78.8 & 117 & 100 & 15 & 1.2 & \\
\hline $05133+0252$ & $\mathrm{AB}$ & 6.9 & 63 & 223 & 25 & 0.3 & & $14426+1929$ & $\mathrm{AC}$ & 135.0 & 309 & 90 & 11 & 1.5 & 1 \\
\hline $05154+3241$ & $\mathrm{AC}$ & 14.2 & 225 & 213 & 18 & 0.5 & & $14497+4843$ & $\mathrm{AB}$ & 2.8 & 45 & 173 & 4 & 0.9 & \\
\hline $05239-0052$ & $\mathrm{AB}$ & 2.9 & 160 & 338 & 2 & 1.6 & 2 & $15185-4753$ & $\mathrm{BC}$ & 22.7 & 129 & 317 & 18 & 0.6 & $\mathrm{O}$ \\
\hline $05248-5219$ & $\mathrm{AC}$ & 38.1 & 288 & 96 & 25 & 0.7 & 1 & $15245+3723$ & $\mathrm{AB}$ & 108.1 & 171 & 103 & 15 & 1.4 & 2 \\
\hline $05301+2933$ & $\mathrm{AC}$ & 15.1 & 352 & 148 & 15 & 0.0 & $\mathrm{mO}$ & $15290-2852$ & $\mathrm{AC}$ & 9.5 & 9 & 66 & 12 & 0.7 & $\mathrm{c} 1$ \\
\hline $05364+2200$ & $\mathrm{AB}$ & 4.0 & 272 & 76 & 3 & 1.6 & & $15332-2429$ & $\mathrm{AB}$ & 9.1 & 301 & 106 & 14 & 0.6 & 2 \\
\hline $05508-3945$ & $\mathrm{AC}$ & 3.7 & 197 & 306 & 19 & 0.3 & 1 & $15387-0847$ & $\mathrm{AB}$ & 11.8 & 189 & 103 & 15 & 0.6 & \\
\hline $06047-4505$ & $\mathrm{AC}$ & 196.2 & 321 & 271 & 6 & 3.0 & $\mathrm{O}$ & $15382+3615$ & $\mathrm{AC}$ & 15.0 & 86 & 142 & 29 & 0.2 & $*$ Oo \\
\hline $06200+2826$ & $\mathrm{AC}$ & 2.9 & 263 & 95 & 7 & 0.8 & 1 & $16035-5747$ & $\mathrm{AC}$ & 11.0 & 243 & 299 & 7 & 1.7 & $\mathrm{w}$ \\
\hline $06298-5014$ & $\mathrm{AC}$ & 12.0 & 312 & 221 & 5 & 2.0 & 3 & $16086-3906$ & $\mathrm{AC}$ & 44.2 & 184 & 93 & 20 & 0.9 & $\mathrm{x}$ \\
\hline $06462+5927$ & $\mathrm{AC}$ & 8.7 & 309 & 99 & 13 & 0.0 & $\mathrm{mO}$ & $16235+3321$ & $\mathrm{AC}$ & 1.0 & 35 & 264 & 13 & 0.4 & \\
\hline $06423-3824$ & $\mathrm{AB}$ & 7.9 & 277 & 166 & 11 & 0.1 & $\mathrm{~m}$ & $16242+3702$ & $\mathrm{AB}$ & 8.3 & 341 & 250 & 10 & 0.8 & \\
\hline $06482+5542$ & BA & 4.6 & 77 & 219 & 23 & 0.2 & & $16238+6142$ & $\mathrm{AB}$ & 1.0 & 352 & 296 & 24 & 0.3 & $\mathrm{X}$ \\
\hline $07031+5410$ & $\mathrm{AB}$ & 9.0 & 66 & 152 & 13 & 0.1 & $\mathrm{~m}$ & $16362+5255$ & $\mathrm{AC}$ & 90.2 & 193 & 81 & 15 & 1.1 & $\mathrm{O}$ \\
\hline $07040-4337$ & $\mathrm{AC}$ & 184.9 & 335 & 79 & 10 & 2.1 & $\mathrm{O}$ & $16579+4722$ & $\mathrm{AC}$ & 112.5 & 262 & 92 & 18 & 1.5 & $\mathrm{O}$ \\
\hline $07148-1529$ & $\mathrm{AC}$ & 15.7 & 2 & 277 & 28 & 0.4 & 10 & $17130-5836$ & $\mathrm{AB}$ & 3.1 & 322 & 279 & 18 & 0.3 & \\
\hline $07171-1202$ & $\mathrm{AC}$ & 15.9 & 242 & 48 & 22 & 0.5 & 1 & $17153-2636$ & $\mathrm{AC}$ & 732.8 & 74 & 83 & 9 & 5.8 & \\
\hline $07201+2159$ & $\mathrm{AB}$ & 5.8 & 220 & 129 & 3 & 2.7 & $\mathrm{w}$ & $17131+5408$ & $\mathrm{AC}$ & 88.5 & 233 & 98 & 8 & 2.4 & \\
\hline
\end{tabular}


Table 1. continued.

\begin{tabular}{|c|c|c|c|c|c|c|c|c|c|c|c|c|c|c|c|}
\hline WDS & SYS & $\rho_{2}{ }^{\prime \prime}$ & $\theta_{2}{ }^{\circ}$ & $\gamma^{\circ}$ & $\epsilon_{\gamma}{ }^{\circ}$ & $\delta^{\prime \prime}$ & note & WDS & SYS & $\rho_{2}{ }^{\prime \prime}$ & $\theta_{2}{ }^{\circ}$ & $\gamma^{\circ}$ & $\epsilon_{\gamma}{ }^{\circ}$ & $\delta^{\prime \prime}$ & note \\
\hline $17190-3459$ & $\mathrm{AC}$ & 32.0 & 138 & 66 & 4 & 5.5 & $\mathrm{w} 1$ & $20078+0924$ & $\mathrm{AB}$ & 3.3 & 340 & 201 & 3 & 1.4 & \\
\hline $17237+3709$ & $\mathrm{AB}$ & 4.1 & 318 & 75 & 8 & 1.0 & $\mathrm{X}$ & $20205-2912$ & $\mathrm{AC}$ & 27.2 & 321 & 71 & 11 & 0.1 & $\mathrm{~m}$ \\
\hline $17322+5511$ & $\mathrm{AB}$ & 62.0 & 311 & 82 & 26 & 0.7 & & $20210-1447$ & $\mathrm{AB}$ & 205.2 & 267 & 94 & 24 & 1.0 & $\mathrm{Xx}$ \\
\hline $17350+6153$ & $\mathrm{AP}$ & 737.4 & 160 & 267 & 2 & 15.0 & c & $20203+3924$ & $\mathrm{AC}$ & 3.4 & 282 & 279 & 8 & 0.7 & 1 \\
\hline $17465+2743$ & $\mathrm{AB}$ & 33.8 & 247 & 48 & 4 & 5.5 & $\mathrm{w}$ & $20587-7025$ & $\mathrm{AB}$ & 6.8 & 113 & 117 & 13 & 0.5 & 2 \\
\hline $17460+3919$ & $\mathrm{AB}$ & 7.9 & 349 & 9 & 29 & 0.2 & & $20591+0418$ & $\mathrm{AC}$ & 10.6 & 68 & 260 & 5 & 1.7 & 1 \\
\hline $17592-3656$ & $\mathrm{AB}$ & 7.5 & 103 & 328 & 7 & 0.9 & & $21022-4300$ & $\mathrm{AB}$ & 57.4 & 73 & 88 & 27 & 0.6 & \\
\hline $18028+7547$ & $\mathrm{AC}$ & 22.9 & 274 & 258 & 11 & 1.9 & $\mathrm{w}$ & $21041-0549$ & $\mathrm{AB}$ & 2.4 & 195 & 139 & 13 & 0.4 & \\
\hline $18002+8000$ & $\mathrm{AB}$ & 19.0 & 232 & 168 & 7 & 1.7 & & $21094-7310$ & $\mathrm{AC}$ & 7.2 & 123 & 235 & 5 & 1.8 & $\mathrm{w}$ \\
\hline $18118+3327$ & $\mathrm{AC}$ & 0.7 & 218 & 57 & 23 & 0.2 & & $21047+0332$ & $\mathrm{AB}$ & 3.4 & 173 & 108 & 4 & 1.5 & \\
\hline $18178+4351$ & $\mathrm{AC}$ & 1.8 & 164 & 269 & 16 & 0.4 & 10 & $21086+3012$ & $\mathrm{AB}$ & 3.4 & 305 & 258 & 9 & 0.6 & \\
\hline $18238+5139$ & $\mathrm{AB}$ & 2.6 & 205 & 95 & 23 & 0.2 & & $21135+0713$ & $\mathrm{AD}$ & 184.1 & 172 & 100 & 8 & 2.8 & c1 \\
\hline $18272+0012$ & $\mathrm{AB}$ & 3.8 & 319 & 97 & 15 & 0.4 & $1 \mathrm{X}$ & $21148+3803$ & $\mathrm{AQ}$ & 89.9 & 184 & 264 & 21 & 1.0 & $\mathrm{wO}$ \\
\hline $18239+5848$ & $\mathrm{AC}$ & 88.9 & 20 & 95 & 29 & 1.0 & $\mathrm{O}$ & $21221+1948$ & $\mathrm{AB}$ & 36.2 & 311 & 96 & 26 & 0.7 & \\
\hline $18338+1744$ & $\mathrm{AC}$ & 1.8 & 275 & 166 & 10 & 0.5 & $1 \mathrm{x}$ & $21223+5734$ & $\mathrm{AP}$ & 83.7 & 192 & 189 & 15 & 0.0 & $\mathrm{mO}$ \\
\hline $18497-7300$ & $\mathrm{AB}$ & 1.9 & 271 & 56 & 8 & 0.5 & & $22024-1658$ & $\mathrm{AB}$ & 3.9 & 246 & 66 & 16 & 0.3 & \\
\hline $18455+0530$ & $\mathrm{AB}$ & 2.5 & 119 & 62 & 8 & 0.6 & 2 & $22038+6438$ & $\mathrm{AB}$ & 7.9 & 276 & 323 & 2 & 2.6 & \\
\hline $18448+3736$ & $\mathrm{AD}$ & 43.7 & 150 & 90 & 27 & 0.6 & & $21582+8252$ & $\mathrm{AB}$ & 13.8 & 67 & 272 & 7 & 1.4 & \\
\hline $18465-0058$ & $\mathrm{AB}$ & 12.7 & 121 & 164 & 13 & 0.6 & & $22375+2356$ & $\mathrm{AC}$ & 5.8 & 194 & 64 & 28 & 0.2 & $\mathrm{O}$ \\
\hline $19021+5216$ & $\mathrm{AB}$ & 5.2 & 299 & 311 & 12 & 0.5 & 2 & $22388-2037$ & $\mathrm{AB}$ & 24.5 & 351 & 311 & 4 & 4.4 & $\mathrm{w}$ \\
\hline $19037+5727$ & $\mathrm{AB}$ & 10.6 & 57 & 42 & 13 & 0.2 & $\mathrm{~m}$ & $22361+7253$ & $\mathrm{AD}$ & 42.2 & 137 & 91 & 20 & 0.9 & $\mathrm{c}$ \\
\hline $19091+3436$ & $\mathrm{AB}$ & 16.1 & 262 & 134 & 6 & 1.7 & & $22397-2820$ & $\mathrm{AB}$ & 86.5 & 159 & 76 & 10 & 0.2 & $\mathrm{~m}$ \\
\hline $19083+5520$ & $\mathrm{AC}$ & 6.5 & 30 & 262 & 9 & 0.8 & 1 & $23069-4331$ & $\mathrm{AC}$ & 159.3 & 292 & 267 & 19 & 0.7 & $\mathrm{O}$ \\
\hline $19313-0207$ & $\mathrm{AB}$ & 1.3 & 63 & 298 & 29 & 0.1 & & $23100+4758$ & $\mathrm{AB}$ & 15.6 & 256 & 62 & 8 & 1.2 & \\
\hline $19407-1618$ & $\mathrm{AC}$ & 45.6 & 42 & 87 & 26 & 0.7 & & $23175+1652$ & $\mathrm{AB}$ & 2.2 & 26 & 228 & 16 & 0.3 & o \\
\hline $20014+1045$ & $\mathrm{AB}$ & 3.8 & 354 & 167 & 5 & 1.0 & & $23191-1328$ & $\mathrm{AB}$ & 12.6 & 351 & 125 & 6 & 1.5 & \\
\hline
\end{tabular}

Notes:

"c": first epoch $\rho, \theta$ are taken from CCDM rather than from WDS;

"w": second epoch position is taken from WDS (no Hipparcos or Tycho identifications);

"*": one of the alternative Hipparcos solutions is accepted;

"1", "2" or "3": Hipparcos or Tycho photocenter positions are computed for primary, secondary or both subsystems of the object, respectively;

"m": Tycho-II proper motion difference is used to compute $\gamma$ instead of $\rho, \theta$-change;

"O", "o": position parameters are corrected for orbital motion in primary, secondary subsystems of the object;

"X", " $\mathrm{x}$ ": uncertainty from unpredictable motion in primary, secondary subsystem is taken into account in $\epsilon_{\gamma}$.

The output sample was thus divided in two halves by the ratio $a_{\text {out }} / a_{\text {in }}$ (the median ratio is $\approx 44$ ). We find that, indeed, the significance of the deviation of $f(\gamma)$ from the uniform one decreases for a "stable" half-sample of objects $\left(a_{\text {out }} / a_{\text {in }}>44\right.$; see Fig. 5$)$ : the $\sim 90^{\circ}$ bins are redistributed into $150^{\circ}-180^{\circ}$ bin. Nevertheless, evidently, the dynamical stability constraint alone is unable to account for the observed $f(\gamma)$.

Angular momentum transfer. The outer subsystem may receive angular momentum and get circularized via the torques from the gaseous material in the system and those from the inner binary (see Artymowicz \& Lubow 1996; Reipurth 2000; Mardling \& Aarseth 2001 and references therein). This process may take place at the earliest stages of the formation of the system. In general, it is characterized by the outward transfer of the angular momentum which makes the inner system more eccentric and the outer more circular. Thus the outer system may become nearly circular, having a degree of hierarchy far above that demanded by pure dynamical stability constraints.
At the current stage, we cannot reach more definitive conclusions about the shape and origin of $f(e)$ for orbits in multiple stars. Larger samples of systems with precisely measured relative proper motions will give a better input information. In this respect, the launch of the GAIA mission of ESA seems to be most promising.

Acknowledgements. Part of this work was supported by the Fellowship of the Belgian Services Fédéraux des Affaires Scientifiques, Techniques and Culturelles, which provided the possibility for the author to work at the Royal Observatory of Belgium. I'm grateful to Dr. A. Tokovinin for his support and encouraging through the progress of this work and to E. van Dessel and Th. Nakos for remarks on the text.

This research made extensive use of Simbad database operated at CDS, Strasbourg, France and of the Digital Sky Survey produced at the Space Telescope Science Institute, USA.

\section{References}

Ambartsumian, V. A. 1937, Soviet Astron. A. J., 14, 207

Artymowicz, P., \& Lubow, S. H. 1996, ApJ, 467, L77 
Brosche, P., Denis-Karafistan, A. I., \& Sinachopoulos, D. 1992, A\&A, 253, 113 (BDS92)

Brosche, P., \& Sinachopoulos, D. 1988, 1989, Bull. Inform. CDS, NN $36 \& 38$

Dommanget, J., \& Nys, O. 1994, Catalogue of the Components of Double, Multiple stars (CCDM) first edition, Comm. Obs. Roy. Belgique, Ser. A, No. 115

Douglass, G. G. 1992, in Complementary Approaches to Double and Multiple Stars Research, ed. H. A. McAlister, \& W. I. Hartkopf, IAU Colloq. 135, ASP Conf. Ser., 32, 311

Duquennoy, A., \& Mayor, M. 1991, A\&A, 248, 485

ESA 1997, Hipparcos and Tycho catalogues, ESA SP-1200

Fabricius, C., \& Makarov, V. V. 2000, A\&AS, 144, 45

Falin, J. L., \& Mignard, F. 1999, A\&AS, 135, 231

Gorti, U., \& Bhatt, H. C. 1996, MNRAS, 283, 566

Harrington, R. S. 1977a, AJ, 82, 753

Harrington, R. S. 1977b, PASP, 89, 400

Høg, E., Fabricius, C., Makarov, V. V., et al. 2000, A\&A, 357, L27
Mardling, R. A., \& Aarseth, S. J. 2001, MNRAS, 321, 398

Palasi, J. 2000, in Birth and Evolution of Binary Stars, Poster Proc. IAU Symp., 200, The Formation of Binary Stars, ed. B. Reipurth, \& H. Zinnecker, 145

Pannunzio, R., Massone, G., \& Morbidelli, R. 1988, A\&A, 203, 388

Patience, J., Ghez, A. M., Reid, I. N., et al. 1998, AJ, 115, 1972

Reipurth, B. 2000, AJ, 120, 3177

Russell, H. N., \& Moore, C. E. 1940, Masses of Stars, Univers. of Chicago

Ruymaekers, G. 1999, Error Assessment of Orbits and Masses of Visual Double Stars by Means of Bootstrap, Ph.D., Katholieke Universiteit Leuven

Tokovinin, A. 1997, A\&A, 124, 75 (MSC)

Tokovinin, A. 1998, Astron. Lett., 24, 178

Worley, C. E., \& Douglass, G. G. 1996, Publ. US Naval. Obs. (WDS) 